\title{
El valor estratégico de la comunicación interna hospitalaria
}

\author{
Pablo Medina Aguerrebere \\ (Neuchâtel, Suiza) \\ Université de Neuchâtel
}

\footnotetext{
Palabras clave

hospital

comunicación institucional comunicación interna comunicación interpersonal

imagen de marca
}

\begin{abstract}
Resumen
En las organizaciones hospitalarias, la comunicación institucional externa e interna constituye una actividad de reciente creación que cada vez se impulsa más debido a varios factores como el desarrollo de las nuevas tecnologías de la comunicación, el surgimiento de los grupos hospitalarios privados, o el interés creciente que la gente manifiesta hacia la salud. La comunicación institucional hospitalaria se ha centrado especialmente en la comunicación externa. Sin embargo, la comunicación interna es cada vez más importante. Por eso, este ensayo basado en una revisión bibliográfica tiene como objetivo evaluar hasta qué punto la comunicación interna pueden ser considerada como una herramienta directiva con valor estratégico. En el ensayo se realiza un análisis bibliográfico sobre el contexto hospitalario, la comunicación institucional en hospitales, y la comunicación interna hospitalaria. La idea que permite concluir este trabajo es que la comunicación interna constituye una verdadera herramienta directiva ya que afecta a todos los empleados del hospital e influye en el funcionamiento de la institución, en la calidad del servicio ofrecido al paciente y en la imagen de marca del hospital.
\end{abstract}




\section{The Strategic Value of Internal Hospital Communication}

\author{
Keywords \\ hospital \\ corporate communication \\ internal communication \\ interpersonal communication \\ brand image
}

\begin{abstract}
In hospital organizations, internal and external corporate communications constitute a recently created activity which is increasingly promoted due to several factors such as the development of new communication technologies, the emergence of private hospital groups, or the growing interest that people have expressed toward health. Corporate communication in hospitals has especially focused on external communication. However, internal communication is increasingly important. Therefore this essay, which is based on a bibliographic study, aims to determine if internal communication can be considered a managerial tool with strategic value. The essay is a bibliographic analysis of the context of hospitals, their corporate communication, and their internal communication. The idea that sets forth the conclusion of this article is that internal communication is a true managerial tool because it affects all hospital employees and influences the hospital's operations, the quality of the service offered to the patient and the hospital's brand image.
\end{abstract}

\section{Cómo citar el artículo}

Medina Aguerrebere, P. (2012). El valor estratégico de la comunicación interna hospitalaria. Revista de Comunicación y Salud, 2(1), pp. 19-28.

DOI: http://doi.org/10.35669/revistadecomunicacionysalud.2012.2(1).19-28

\section{Introducción}

El envejecimiento de la población, el desarrollo de la sanidad privada, la implantación de las nuevas tecnologías de la comunicación en el ámbito hospitalario y el impacto de internet como fuente de información médica son algunos de los aspectos que más influyen en el funcionamiento del sistema hospitalario actual. En este contexto, cada vez más organizaciones hospitalarias comienzan a considerar a la comunicación institucional interna y externa como una herramienta estratégica capaz de influir de forma positiva en el buen funcionamiento del hospital. De ahí, que cada vez más hospitales implanten departamentos de comunicación institucional, cuyo objetivo es la gestión de la imagen del hospital así como de las relaciones de dicha organización con sus stakeholders principales (pacientes, empleados, autoridades sanitarias, etc.). Dichas organizaciones adoptan una visión global del concepto de comunicación institucional en el que se da cabida a las actividades externas (comunicación con públicos externos como los pacientes, los periodistas, etc.) así como las actividades internas (comunicación con los empleados, etc.). 
La comunicación institucional se centra especialmente en las relaciones externas del hospital, lo cual da lugar a iniciativas como las relaciones con los medios de comunicación (envío de comunicados, organización de ruedas de prensa, etc.), el desarrollo de una página web, la celebración de eventos externos (conferencias, cursos, etc.) e incluso la ejecución de ciertas labores de marketing (difusión de dípticos, campañas de publicidad, etc.). En este contexto tan marcado por el rol de la comunicación externa, hay que plantearse la siguiente pregunta: ¿se puede considerar a la comunicación interna hospitalaria como una herramienta estratégica de dirección? Para intentar responder a esta pregunta, el documento analiza, en primer lugar, el contexto hospitalario; en segundo lugar, el rol de la comunicación institucional en los hospitales; y en tercer lugar, antes de las conclusiones, el carácter estratégico de la comunicación interna hospitalaria.

\section{El contexto hospitalario}

Los ciudadanos se preocupan cada vez más por su salud (Fisac, 2006), lo cual, en cierto modo, se deriva del interés creciente que genera este tema en la opinión pública. Así, por un lado, en los últimos años distintas organizaciones que no pertenecen al ámbito sanitario (empresas de alimentación, de turismo, etc.) han desarrollado productos y servicios orientados a la promoción de la salud del ciudadano; y por otro lado, según el informe publicado por el Observatorio de la Comunicación Científica (2008), el número de artículos que los periódicos El País, El Mundo, $A B C$, La Vanguardia y El Periódico de Cataluña han dedicado a este tema se ha duplicado en los últimos diez años (4.079 artículos publicados en 1997, y 9.247 en 2006). Sin embargo, la salud no es sólo un elemento informativo y una preocupación, sino también un derecho. Así, el Pacto Internacional de Derechos Económicos, Sociales y Culturales, en su artículo doce, apartado primero, subraya que "los Estados Partes en el presente Pacto reconocen el derecho de toda persona al disfrute del más alto nivel posible de salud física y mental" (Organización de las Naciones Unidas, 1976).

En este contexto tan marcado por la importancia creciente de la salud, los hospitales se han convertido en instituciones que asumen una misión de gran valor social. La Real Academia Española (2001) define a esta institución, en su primera acepción, como el "establecimiento destinado al diagnóstico y tratamiento de enfermos, donde se practican también la investigación y la enseñanza". A estas tres funciones, se podría añadir una cuarta: la promoción de los hábitos de vida saludables entre la población. Sin embargo, la gestión de los hospitales y de las distintas funciones que asumen estas organizaciones no resulta fácil. Todos los hospitales son instituciones complejas de gestionar (Ladrón de Guevara Portugal, 2005), especialmente debido a tres razones. En primer lugar, los hospitales son sistemas complejos formados por varios subsistemas interrelacionados que necesitan trabajar de forma coordinada y conjunta (Wright, Sparks y O'Hair, 2008). En segundo lugar, en los hospitales trabaja un gran número de personas, todas ellas especializadas en ámbitos científicos y profesionales muy distintos entre sí. Y en tercer lugar, el paciente está presente durante la prestación del servicio médico (consultas, intervención quirúrgica, pruebas diagnósticas, etc.), lo cual dificulta ciertos procesos de management. 
Estas son algunas de las características que definen el contexto hospitalario, y que determinan el trabajo de los principales protagonistas del hospital, es decir, el personal médico, personal de enfermería, paciente, familiares del paciente, directivos de salud y empleados que realizan labores administrativas o de mantenimiento. Todos los profesionales del hospital tienen como objetivo prioritario mejorar la salud de las personas (Expósito Hernández et al. 2002), para lo cual se hace necesario considerar ciertos elementos del contexto hospitalario, como por ejemplo el aspecto informativo (transmisión de información entre los empleados del hospital, y entre dichos empleados y el paciente), el aspecto social y emocional (connotaciones vitales de la enfermedad y de los tratamientos médicos), y el aspecto cultural (diferencias idiomáticas, culturales y religiosas entre el profesional de la salud y el paciente). Dichos elementos intangibles influyen en el servicio de salud ofrecido al paciente así como en el funcionamiento del propio hospital. Por ello, los hospitales necesitan conceder más importancia a la comunicación institucional interna y externa como vector de dichos elementos intangibles.

\section{La comunicación institucional en el hospital}

La gestión profesional de la comunicación institucional interna y externa en los hospitales es una actividad reciente que se ha impulsado en los últimos años gracias al desarrollo, dentro del hospital, de las distintas labores de gestión y de las nuevas tecnologías de la comunicación y de la información. El impacto social de estas instituciones, el desarrollo de la sanidad privada y de la competencia internacional, el valor creciente asignado a los intangibles y el desarrollo de internet como fuente de información médica son algunos de los factores que hacen cada vez más necesario que cada hospital desarrolle su arquitectura de marca, es decir, su identidad y valores, misión, visión, cultura, e imagen.

Los hospitales difunden su identidad corporativa a través de varias acciones, entre las que destacan los momentos de contacto con el paciente, el principal público externo de esta institución. Cuando el cliente entra en contacto con la marca, éste juzga si la promesa de la organización es verdadera o no (Quirke, 2000), de ahí la importancia de que el comportamiento del personal médico que tiene contacto directo con el paciente, así como el contenido de las distintas herramientas de comunicación usadas por el hospital (página web, revista corporativa, etc.) se ajusten a la identidad corporativa de la organización y a sus valores. "Los directivos necesitan ser completamente conscientes del impacto tremendo de la identidad, de la imagen y de la reputación y deben aprender a gestionar estos recursos" (Argenti, 2003: 76). Esta actitud de los directivos da lugar a varias acciones, como por ejemplo, la organización de cursos para que los empleados conozcan mejor la arquitectura de marca del hospital.

Además de la identidad, los hospitales necesitan definir el resto de elementos que conforman la arquitectura de marca, los cuales pueden influenciar positivamente en el funcionamiento del hospital. Así, la misión ayuda a la organización a mejorar el conocimiento sobre el público, a señalar las prioridades de la organización y a establecer el tono de la comunicación corporativa (Parsons, 2001). Por su parte, la visión influye en la motivación de los empleados del hospital. "La misión describe los medios, la visión describe el objetivo y establece una declaración cautivadora de lo que la organización intenta conseguir" (Barret, 2003: 83). 
Respecto a la cultura, se puede señalar que ésta ayuda al hospital a establecer protocolos de funcionamiento coherentes con la forma de ser de los empleados. Siguiendo a Barret (2003), la cultura de la institución está constituida por creencias, que a su vez elaboran un modelo mental que determina la percepción que los empleados de la empresa tienen del mundo. Por último, la imagen de marca desempeña una labor esencial ya que ayuda a la organización hospitalaria a mantener un contacto satisfactorio y durable con su sociedad de referencia y con sus stakeholders principales. Según Costa (2001: 68-77), la imagen de marca de una empresa tiene quince funciones: destacar la identidad, definir la cultura, construir la personalidad, reforzar el "espíritu de cuerpo" o trabajo en equipo, atraer a los trabajadores, motivar a los inversores, evitar las crisis, impulsar los nuevos productos, relanzar a la empresa, generar una opinión pública favorable, reducir los mensajes involuntarios, optimizar las inversiones en comunicación, acumular reputación, atraer a los clientes, e inventar el futuro.

La organización hospitalaria puede y debe apostar por el desarrollo de una arquitectura de marca que siente las bases de su política de comunicación institucional. A pesar de las dificultades que esta actividad encuentra en el ámbito hospitalario (escasa tradición comunicativa, contexto legal, connotaciones emocionales del servicio hospitalario, etc.), dichas organizaciones deben apostar por la implantación de la comunicación institucional interna y externa como actividad estratégica ya que esta decisión les puede aportar varios beneficios, entre los que destacan tres.

En primer lugar, la comunicación institucional ayuda a crear lazos sociales entre los empleados y a evitar conflictos (Décaudin, Igalens y Waller, 2006), lo cual es importante dado el gran número de trabajadores que tienen los hospitales y el contexto cambiante del sector sanitario. La comunicación interpersonal entre los empleados del hospital constituye una de las acciones de comunicación interna más importantes que tienen lugar en el hospital. En segundo lugar, la comunicación influye en la satisfacción del paciente. Así, la comunicación interpersonal mantenida entre el médico y el paciente puede influir en los resultados físicos y psicológicos que obtiene el paciente con el tratamiento médico (Wright, Sparks y O'Hair, 2008). Este tipo de comunicación interpersonal médico-paciente, aunque tiene lugar en las instalaciones del hospital, se considera como una acción de comunicación externa, dado que el paciente es un público externo. $Y$ en tercer lugar, la comunicación institucional ayuda al hospital a gestionar algunos de los problemas que debe afrontar con sus distintos stakeholders (pacientes, medios de comunicación, autoridades sanitarias, etc.). Así, la comunicación es un proceso relacional global que va más allá del mero intercambio de elementos informativos (Condit, 2005) y que influye positivamente en el funcionamiento de las instituciones.

\section{El carácter estratégico de la comunicación interna hospitalaria}

Los intangibles asociados al acto médico y sanitario en general (connotaciones sociales, culturales, emocionales, etc.), el número elevado de empleados y de perfiles profesionales distintos que conviven en los hospitales, así como la necesidad de trabajar de modo coordinado para ofrecer un servicio de calidad a los pacientes -quienes asisten de modo presencial a la prestación del mismo- son algunos de los factores que convierten a la comunicación interna 
en la vertiente de la comunicación institucional más importante del hospital. Aunque existen muchas formas de definir a la comunicación interna, la siguiente definición resulta especialmente apropiada para el ámbito hospitalario:

"Tipo de comunicación que favorece o promueve relaciones eficientes entre las personas que configuran los públicos internos de una organización, y que genera la confianza necesaria para coordinar adecuada y responsablemente todos los recursos disponibles en la consecución de la misión compartida. Su objetivo es mejorar la calidad del trabajo de la organización y llevar a cabo con mayor perfección la realización de su misión" (La Porte, 2001: 43).

En el ámbito hospitalario, la comunicación interna adquiere un valor estratégico ya que influye de modo positivo en los empleados (público interno), en los pacientes (público externo) y en la institución en su conjunto. La comunicación interna influye positivamente en los empleados ya que, por un lado, les ofrece información sobre distintos ámbitos que les ayudan a ser más eficaces en su trabajo, y por otro lado, porque contribuye a instaurar un ambiente de trabajo agradable donde la cohesión social prima sobre el antagonismo. Gracias a las acciones de comunicación interna, los empleados reciben información sobre varios temas, como por ejemplo la arquitectura de marca de la organización, las estrategias y objetivos del hospital, o las opciones de formación en habilidades profesionales. Para que la estrategia de la empresa pueda llevarse a la práctica, resulta esencial que los trabajadores conozcan al detalle dicha estrategia (Quirke, 2000), y para informarles de modo eficaz sobre la misma así como sobre los objetivos de la empresa, la página web constituye una herramienta adecuada (Holtz, 2004). Así, por ejemplo, en España el $45 \%$ de los empleados considera tener un conocimiento alto sobre los objetivos de la organización (Observatorio de Comunicación Interna e Identidad Corporativa, 2006).

Además de la difusión de información a los empleados, la comunicación interna contribuye a crear un ambiente de trabajo agradable caracterizado por la cohesión, el trabajo en equipo y la ausencia de antagonismos, algo que resulta esencial en el hospital dado el número de trabajadores de los que dispone, la necesaria coordinación de procesos y las condiciones de estrés y urgencia en las que muchas veces se realizan dichos trabajos. "La información consolida la alineación entre capital humano y estrategia, contribuye al entendimiento y fortalece la confianza requerida para la aceptación del acuerdo laboral" (Davenport, 2000: 143). Este rol de la información como creadora de lazos sociales resulta especialmente importante durante los procesos de cambio o durante la gestión de crisis dentro del hospital. La comunicación interna constituye una herramienta eficaz que ayuda a los empleados y a la organización a adaptarse a las nuevas situaciones (Elías y Mascaray, 1998).

La comunicación interna no solo influye positivamente en los empleados, sino que también influye en el funcionamiento de la institución hospitalaria en su conjunto ya que ayuda a la dirección de la misma a obtener información sobre los públicos internos (empleados) y los públicos externos (especialmente, los pacientes). Los hospitales se interesan por las necesidades clínicas del paciente, pero también por las necesidades no clínicas, ya que así se puede lograr una ventaja competitiva (Halley, 2007). Además, la comunicación interna permite dina- 
mizar el funcionamiento de la institución en el sentido de que ayuda a los empleados a comprender el impacto estratégico de su trabajo y la necesidad de alcanzar los objetivos globales de la organización. La verdadera utilidad de la comunicación interna reside en su capacidad para ayudar a los empleados a convertir la estrategia en acción (Quirke, 2000).

Por otro lado, hay que señalar que gracias a la comunicación interna, los hospitales pueden difundir entre sus empleados la información concerniente a la arquitectura de marca de la organización. "La promoción de la marca a nivel interno es también importante a la hora de construir la moral y de crear un lugar de trabajo donde los empleados se comprometan con su profesión" (Argenti, 2003: 13). En el ámbito hospitalario, resulta esencial que los empleados conozcan la arquitectura de marca de la organización ya que en estas instituciones existe un número elevado de empleados que tiene contacto directo con el paciente y con sus familiares (públicos externos). Así, la comunicación interpersonal entre los empleados (comunicación interna) y la comunicación interpersonal entre los empleados y los pacientes (comunicación externa) se convierten en herramientas que permiten a la institución difundir su identidad entre sus stakeholders más importantes (empleados y pacientes). Por último, hay que indicar que los directivos hospitalarios de España consideran que los aspectos que más influyen en la mejora del funcionamiento del hospital son el trato de médicos o enfermeras, la atención a pacientes, y la imagen de comodidad o calidad (Esade e Inforpress, 2004), lo cual permite realzar de nuevo la importancia estratégica de la comunicación interpersonal entre empleados así como la que tiene lugar entre empleados y pacientes.

Por último, es necesario recalcar que la comunicación interna influye también de modo positivo en el funcionamiento organizacional del hospital, especialmente en lo que concierne a la gestion de los procesos internos, la optimización de los costes, y la atención al paciente. En lo que se refiere a los procesos internos, la información tiene un valor estratégico para los ejecutivos de salud (Pérez Sáez, 2002), de hecho muchos proyectos de comunicación interna tiene su origen en las necesidades de la dirección general de la empresa (Beal y Lestocart, 2003), las cuales conciernen, en la mayor parte de los casos, a la optimización de los procesos internos del hospital. En cuanto a la optimización de costes, según Parsons (2001), la comunicación interna asume como principal objetivo la generación del diálogo dentro de la institución, lo cual ayuda a dicha organización a mejorar la coordinación interdepartamental y, por tanto, los costes hospitalarios. Y por último, respecto a la atención al paciente, según Asenjo (2002), la calidad asistencial es el resultado de la combinación de dos elementos: el conocimiento y la tecnología por un lado, y el aspecto humanitario (trato al enfermo, información, comunicación, etc.), por otro. Así, la relación directa entre la gestión y la comunicación interna en los hospitales es una realidad. De hecho, según Mucchielli (2005: 184), la gestión es "el arte de resolver, a través de distintas formas de comunicación interna, los problemas organizacionales comunes y recurrentes que se presentan a todo responsable".

\section{Conclusiones}

En la introducción, se señalaba que el objetivo de este trabajo era saber si la comunicación interna hospitalaria puede ser considerada como una herramienta estratégica de dirección. Des- 
pués de haber analizado el contexto hospitalario, el rol de la comunicación institucional en hospitales y el impacto de la comunicación interna en dichas organizaciones, se puede afirmar que la respuesta a la pregunta planteada en la introducción es afirmativa debido a dos razones.

En primer lugar, la comunicación interna es una actividad que persigue diferentes objetivos, que recurre a distintas estrategias y soportes de comunicación (intranet, revista corporativa, etc.), que influye en varios aspectos de la organización (procesos de gestión, procesos médicos, etc.) y que concierne a todos los empleados, por ello requiere una gestión estratégica y directiva que permita orientar esta actividad hacia la consecución de las metas globales que persigue el hospital. Y en segundo lugar, hay que considerar a la comunicación interna como una herramienta estratégica de dirección porque muchas de las iniciativas que se emprenden bajo el marco de esta actividad influyen en la calidad del servicio médico global que los hospitales ofrecen a los pacientes. Y por tanto, la gestión correcta de esta actividad influye en la satisfacción del paciente, lo cual, a su vez, influye en la imagen de marca y en la reputación de la organización.

Por último, hay que señalar que el carácter estratégico de la comunicación interna justifica la creación, dentro del hospital, de un departamento de comunicación interna que asuma esta actividad de una forma autónoma y protocolizada (creación de protocolos y de un plan oficial de comunicación interna) en la que la colaboración con los demás departamentos de la institución, por un lado, y la formación del profesional de la salud en el ámbito de la comunicación interna, por otro, constituyan sus dos principales áreas de actividad.

Referencias

Argenti, Paul. (2003). Corporate communication. Boston: McGraw-Hill//rwin.

Asenjo, Miguel Ángel. (2002). Las claves de la gestión hospitalaria (2ª Ed.). Barcelona: Gestión 2000, Cop.

Barret, Richard. (2003). Libérer l'âme de l'entreprise. Bâtir une organisation visionnaire guidée par les valeurs. Bruxelles; Paris: De Boeck.

Beal, Jean-Pierre y Lestocart, Pierre-André. (2003). Entre gestion et marketing: la communication interne. Condé-sur-Noireau: Les Éditions Demos.

Condit, Celeste. (2005). Communication as relationality. En Shepherd, Gregory; John, Jeffrey y Striphas, Ted. Communication as... perspectives on theory. Thousand Oaks, California: Sage Publications.

Costa, Joan. (2001). Imagen corporativa en el siglo XXI (1ª Ed.). Buenos Aires: La Crujía.

Davenport, Thomas. (2000). Capital humano: creando ventajas competitivas a través de las personas. Barcelona: Gestión 2000.

Décaudin, Jean-Marc; Igalens, Jacques y Waller, Stéphane. (2006). La communication interne: stratégies et techniques. Paris: Dunod. 
Elías, Joan y Mascaray, José. (1998). Más allá de la comunicación interna: diez estrategias para la implantación de valores y la conquista del comportamiento espontáneo de los empleados. Barcelona: Gestión 2000.

Esade e Inforpress (2004). La comunicación en el entorno hospitalario. Primer estudio sobre la comunicación en los centros hospitalarios españoles. Madrid: Esade e Inforpress.

Expósito Hernández, José; Gallo Vallejo, Francisco Javier; García Cubillo, María; Márquez Calderón, Soledad; Martínez Tapia, Jesús y Pérez Sáez, Francisco. (2002). La verdadera experiencia de gestión. En Expósito Hernández, José. El trabajo de gestión desde una dirección médica: los profesionales, las prácticas clínicas y los gestores. Granada: Hospital Universitario Virgen de la Nieves.

Fisac, Vicente. (2006). La comunicación de los avances terapéuticos: principios éticos y responsabilidad del periodista. En De Quiroga, Santiago. Comunicar es salud. Madrid: Prentice Hall, Financial Times.

Halley, Marc. (2007). The primary care-market share connection: how hospitals achieve competitive advantage. Chicago: Health Administration Press.

Holtz, Shel. (2004). Corporate conversations: a guide to crafting effective and appropriated internal communications. New York: Amacom.

Ladrón de Guevara Portugal, José Manuel. (2005). Los hospitales en el umbral del siglo XXI: el desafío de ofrecer a la sociedad un hospital cada día mejor. Santander: Academia de Medicina de Cantabria.

La Porte, José María. (2001). Entusiasmar a la propia institución: gestión y comunicación interna en las organizaciones sin ánimo de lucro. Madrid: Ediciones Internacionales Universitarias.

Mucchielli, Alex. (2005). Information et communication interne: étude des communications. Pour de nouveaux audits (2ème Éd.). Paris: Armand Colin.

Observatorio de Comunicación Interna e Identidad Corporativa. (2006). Marca interna y mandos intermedios: claves para la comunicación interna como estrategia empresarial. IV estudio de la comunicación interna en las empresas españolas. Madrid: Observatorio de Comunicación Interna e Identidad Corporativa.

Observatorio de la Comunicación Científica. (2008). Medicina y salud en la prensa diaria. Informe Quiral 10 años. Barcelona: Noclay.

Organización de las Naciones Unidas. (1976). Pacto Internacional de Derechos Económicos, Sociales y Culturales. Disponible en http://www2.ohchr.org/spanish/law/cescr.htm. Recuperado el 10 de septiembre de 2011.

Parsons, Patricia. (2001). Beyond persuasion: the healthcare manager's guide to strategic 
communication (1st Ed.). Chicago: Health Administration Press.

Pérez Sáez, Francisco. (2002). La información asistencial. Instrumento para qué y para quién. En Expósito Hernández, José. El trabajo de gestión desde una dirección médica: los profesionales, las prácticas clínicas y los gestores. Granada: Hospital Universitario Virgen de la Nieves.

Quirke, Bill (2000). Making the connections: using internal communication to turn strategy into action. Aldershot: Gower.

Real Academia Española (2001). Diccionario de la lengua española (22ª Ed.) Madrid: EspasaCalpe.

Wright, Kevin; Sparks, Lisa y O'Hair, Dan (2008). Health communication in the 21st century. Malden: Blackwell Pub. 